Euskal ikerketen aldizkaria | Revue d'études basques |

Revista de estudios vascos | Basque studies review

$8 \mid 2003$

Numéro VIII

\title{
Nommer, définir des faits linguistiques : antipassif et sujet, deux exemples en basque
}

Jean-Baptiste Coyos

\section{OpenEdition \\ Journals}

Édition électronique

URL : http://journals.openedition.org/lapurdum/939

DOI : 10.4000/lapurdum.939

ISSN : 1965-0655

Éditeur

IKER

Édition imprimée

Date de publication : 1 novembre 2003

Pagination : 123-138

ISBN : 9782867813436

ISSN : $1273-3830$

Référence électronique

Jean-Baptiste Coyos, « Nommer, définir des faits linguistiques : antipassif et sujet, deux exemples en basque », Lapurdum [En ligne], 8 | 2003, mis en ligne le 01 mai 2009, consulté le 30 avril 2019. URL : http://journals.openedition.org/lapurdum/939; DOI : 10.4000/lapurdum.939 


\title{
Nommer, définir des faits linguistiques : antipassif et sujet, deux exemples en basque
}

\author{
Jean-Baptiste / Battittu COYOS
}

IKER UMR 5478 CNRS

\begin{abstract}
LABURPENA : Lan honetan aztertzen dugu nola antipasibo eta subjektu nozioak irabiliak izan diren euskal ikerketetan. Lehen hitza berri berria da hizkuntzalaritzaren historian, bigarrena Aro helenistikotik etortzen zaigu. Erakusten dugu zergatik antipasibo nozioa ez dugu erabiltzen $R$. de Rijken adieraren arabera. Subjektu hitzaz, gure kuadro teorikoan beharrezkoa ez dela azaltzen dugu euskara bezala hizkuntza ergatibo bat zehazteko.
\end{abstract}

\section{INTRODUCTION : QUELQUES PRINCIPES ET PRECAUTIONS METHODOLOGIQUES}

Cette contribution ne s'appuie pas sur une réflexion épistémologique approfondie. On ne cherche pas ici à statuer sur les principes, les concepts, les méthodes de telle ou telle théorie linguistique ou des sciences du langage en général. Il s'agit à travers de faits linguistiques du basque de réfléchir à deux notions utilisées en linguistique, celle d'antipassif et celle de sujet, en observant succinctement comment elles sont appliquées à la langue basque et, dans la mesure où cela paraît utile, de prendre position.

Cette étude sans prétention m'a été suggérée par la lecture du dernier article de Rudolf P. G. de Rijk, "L'antipassif et l'hypothèse de Levin », paru dans le précédent numéro de cette même revue Lapurdum. Plutôt qu'une réponse, on voudra voir dans ce texte un modeste hommage à ce bascologue hollandais récemment disparu ${ }^{1}$. C'est aussi l'accumulation de lectures de points de vue divers sur la langue basque qui m'a incité à écrire ces lignes.

On peut prendre comme point de départ de la réflexion cette citation de Gilbert Lazard : «L'objectif principal de la linguistique, entendue comme science de la langue (par opposition à la parole...) est la découverte d'invariants interlangues, c'est-à-dire de relations plus ou moins abstraites qui se vérifient dans les langues les plus diverses. C'est donc par la recherche typologique que l'on peut espérer les atteindre. Celle-ci comporte deux étapes : d'abord la description des langues, puis leur comparaison... Les catégories étant propres à chaque langue, ne coïncident généralement pas d'une langue à l'autre... Il faut donc recourir au contenu sémantique, qui est ce que les langues ont en commun,... [et] construire des cadres conceptuels qui serviront de base de comparaison... ces grilles conceptuelles... sont néanmoins logiquement arbitraires et provisoires. Ce sont des hypothèses de travail utilisées comme des instruments pour la découverte des relations invariantes » $(2000: 447)^{2}$.

\footnotetext{
${ }^{1}$ Décédé le 15 juin 2003, Rudolf P. G. de Rijk était membre d'honneur de l'Académie de la langue basque, Docteur « honoris causa » de l'Université du Pays basque. Il fut le pionner dans les années 1960 de l'application du modèle générativiste à la langue basque. Un choix de ses écrits sur la langue basque, De Lingua Vasconum : selecting writings a été publié en 1998 dans l'Anuario del Seminario de Filología Vasca "Julio de Urquijo", supplément XLIII. Ses Cours de grammaire basque pour linguistes en 27 chapitres devraient paraître prochainement.

${ }^{2}$ Ce linguiste, qui appartient au courant structuraliste fonctionnaliste, a un objectif au premier abord pas bien éloigné d'un Jean-Yves Pollock qui, dans Langage et cognition, présente « les outils conceptuels fondamentaux d'un modèle [le programme minimaliste de la grammaire
} 
Un autre principe est que "dans toutes les sciences, l'objectivité d'une théorie est assurée par sa confrontation avec les données empiriques... On sait bien qu'il n'y a pas de faits bruts... Le fait scientifique est toujours construit sur la base d'une théorie » (Lazard, $2001:$ 12). On a alors un cercle vicieux auquel on peut échapper à condition que «l'outillage expérimental jouisse d'une indépendance logique à l'égard des propositions soumises au test » (Milner, 1989 : 127, cité par Lazard, $2001: 12$ ).

C'est à partir de tels principes, en s'appuyant sur des données morphologiques, syntaxiques du basque et évidemment, on l'a vu, les contenus sémantiques véhiculés que je vais tenter de discuter deux notions qui sont appliquées à cette langue, celle récente d'antipassif d'abord, puis celle issue de la tradition grammaticale de l'Antiquité de sujet.

\section{LA NOTION D'ANTIPASSIF ET LA LANGUE BASQUE}

Cette première notion est particulièrement intéressante car elle est apparue il y a moins de trente ans dans la terminologie linguistique, alors qu'en langue basque on peut lui faire correspondre une construction ancienne, clairement identifiable, analysable, avec un contenu sémantique plus ou moins spécifique. Cette construction avait été repérée en basque, mais les grammaires ne lui avaient accordé aucune ou très peu d'importance.

\subsection{Création du terme antipassif et définition du concept}

Le terme antipassif a été forgé («coined»), selon Dixon, fin 1968 par le linguiste américain Michael Silverstein à propos du dyirbal lors d'une conférence de ce même Dixon sur les langues d'Australie (1994 : 149, note 9). Selon de Rijk, le terme a été utilisé pour la première fois par Silverstein lors d'un exposé au printemps 1969 et par écrit en 1972 à propos de la langue amérindienne chinook ${ }^{3}$. La construction du terme est transparente. Citons Silverstein d'après de Rijk : " I have termed this -ki- form the ANTIPASSIVE construction, playing upon its inverse equivalence to a passive of accusative languages, because the sense is clearly equivalent to a transitive, though the form is intransitive » (2002 : 297). L'antipassif est donc une construction symétrique du passif mais repérée dans les langues ergatives.

Afin de pouvoir maintenant discuter ce que de Rijk entend par antipassif en basque et expliquer pourquoi il me semble plus adapté d'appliquer le terme à une autre construction que celle qu'il a retenue, il est nécessaire de donner la seule définition de l'antipassif que celui-ci a pu relever dans les dictionnaires de linguistique qu'il a examinés ${ }^{4}$. Il s'agit d'une définition donnée par Dixon,

générative] qui vise à isoler, à travers l'étude détaillée d'un nombre de langues aussi grand que possible, les propriétés universelles d'un module particulier de l'appareil conceptuel humain, $s a$ faculté de langage » (1997: $4^{\text {ème }}$ de couverture). Je ne m'engagerai pas sur l'affirmation suivante de Lazard qui conclut l'article cité en disant : «le but du linguiste est de découvrir, non d'expliquer; l'explication relève d'autres disciplines (psycho-linguistique, sciences cognitives, etc.) » (2000: 459). Ici les deux courants théoriques divergent puisque Pollock met le programme minimaliste «en perspective... du point de vue des propriétés linguistiques et psychologiques dégagées »(1997: ibid., c'est moi qui souligne); "la linguistique est une branche de la psychologie cognitive puisque son objet, le savoir linguistique d'individus particuliers, est une réalité psychologique et donc, neuro-physiologique » (1997:1).

${ }^{3}$ On me pardonnera de ne pas avoir été vérifier à la source.

${ }^{4}$ Aucun dictionnaire de sciences du langage en français ne mentionne l'antipassif. 
justement, dans l'International Encyclopedia of Linguistics $(1992, \mathrm{I}: 136)^{5}:$ " the ANTIPASSIVE derivation : deep A (c.-à-d. le sujet transitif) becomes surface $\mathrm{S}$ (c.-à-d. le sujet intransitif), deep $O$ (c.-à-d. l'objet direct) is marked by an oblique case (dative, locative, or instrumental, in different languages), and the verb bears an antipassive derivational suffix » (220: 295, les incises sont de de Rijk). Si, avec ce dernier, on peut noter le caractère "circulaire" de l'expression « antipassive derivational suffix » pour définir justement l'antipassif, on va voir que celui-ci n'a pas retenu la nécessité d'un affixe supplémentaire par rapport à la diathèse non marquée pour former une construction antipassive.

On sait que c'est Georges Rebuschi qui a mis à l'honneur l'antipassif dans les études basques avec l'article de 1979, "Autour du passif et de l'antipassif en basque biscayen (sous-dialecte d'Oñate) ». Des constructions du type décrit par Rebuschi avaient été signalées très épisodiquement, du bout des lèvres si l'on peut dire par quelques bascologues, mais c'est «l'abbé Jean Larrasquet qui, à ma connaissance, est le seul en 1934 dans son ouvrage intitulé Le Basque Souletin Nord-Oriental à avoir donné dans les listes des formes du verbe ükhen « avoir », à partir de la page 165, les formes antipassives comme ükhénik nük qu'il traduit par «j'ai fini d'avoir (de recevoir)» et qu'il nomme parfait pour cette forme, plusque-parfait ou futur antérieur pour d'autres »(Coyos, 2002: 289). Il faut d'ailleurs noter que « beaucoup de chercheurs, pas tous, qui ont étudié la langue basque mettent plus ou moins nettement en doute l'existence même de diathèses, de parcours diathétiques diversifiés en basque » (ibid., 287). L'Académie de la langue basque n'est pas en reste puisqu'il est dit dans Euskal gramatika Lehen urratsak-I : «Euskaraz ez dugu diatesi-rik (erderetako pasiboa) (1991: 222). Quant à l'antipassif, parcours diathétique conforme aux traits typologiques attendus d'une langue dite ergative, il n'est pas évoqué.

\subsection{L'antipassif en basque selon Rudolf $P$. G. de Rijk relève-t-il d'un parcours diathétique marqué ? Arguments en sa défaveur}

Venons-en maintenant au point de vue développé par de Rijk concernant ce qui relève de l'antipassif en basque. Selon lui les constructions citées par Rebuschi dans ses travaux successifs et tenues pour antipassives n'en sont pas. C'est le cas d'une phrase comme Umea $a_{\mathrm{ABS}}$ liburua $_{\mathrm{ABS}}$ leiuta dao (ABS pour absolutif), qu'on pourrait traduire pas «l'enfant se trouve ayant lu le livre », car non conforme à la définition donnée par Dixon de l'antipassif où « le syntagme absolutif figurant dans la structure ergative est censé se dégrader en cas oblique ; dans l'exemple rebuschien, par contre, il persiste tel quel » (de Rijk, 2002 : 298). Effectivement liburua reste à l'absolutif comme dans la phrase active ${\text { correspondante } \text { Umeak }_{\mathrm{ERG}} \text { liburua }}_{\mathrm{ABS}}$ leiu dau «l'enfant a lu le livre » (ERG pour ergatif). De même Haurrak $k_{\mathrm{ABS}} z o p a_{\mathrm{ABS}}$ janik dira, exemple que j'ai pu donner et qu'il cite, ne relève pas du parcours diathétique antipassif selon de Rijk.

Celui-ci demande donc à Rebuschi et moi-même de «changer de terme en inventant une autre désignation, que ce soit parapassif, quasipassif, métapassif, ou je ne sais quoi, mais non antipassif, car ce terme-là, déjà breveté, ne se trouve plus disponible (2002 : 299) ». Voici un des quatorze exemples en paires qu'il propose alors, considérant que «l'emploi de l'auxiliaire intransitif dans le second membre par opposition à l'auxiliaire transitif du premier s'interprète sans peine comme un marqueur de l'antipassif » (2002: 299) : Hiruek $k_{\mathrm{ERG}}$ mintzatzen dute euskara / Hiruak $k_{\mathrm{ABS}}$ mintzatzen dira euskaraz « les trois parlent basque ».

\footnotetext{
${ }^{5}$ William Bright, International Encyclopedia of Linguistics, New York / Oxford.
} 
De Rijk convient qu'on pourrait classer cette alternance de constructions propre à certains verbes comme « un phénomène purement lexical» (2002 : $3003)^{6}$, mais retient ces faits de synchronie pour « postuler un véritable procédé antipassif pour le basque ancien, disons du premier millénaire » (2002: 304). Bien incapable de discuter cette hypothèse, je voudrais souligner en quoi cette alternance ne relève pas d'une transformation de diathèse, en quoi la construction intransitive type Hiruak mintzatzen dira euskaraz n'est pas conforme à la définition de Dixon si tant est que cette définition doit être considérée comme la définition de l'antipassif, c'est-à-dire une proposition qui fait connaître une fois pour toutes la compréhension et l'extension de cette notion.

- Tout d'abord, il n'y a pas véritablement de changement de diathèse entre Hiruek $_{\mathrm{ERG}}$ mintzatzen dute euskara ${ }_{\mathrm{ABS}}$ et Hiruak ${ }_{\mathrm{ABS}}$ mintzatzen dira euskaraz INST $_{\mathrm{IN}}$ en ce sens que le point de départ et d'arrivée sont les mêmes; on part de l'argument à rôle sémantique agentif vers l'argument à rôle sémantique non agentif. La relation entre les deux entités impliquées dans le comportement est parcourue dans le même sens. Mais on peut en dire autant des exemples que de Rijk remet en cause. En tout cas, en synchronie, on ne peut pas dire qu'une des constructions est issue de l'autre : mintzatu «parler» a deux constructions possibles à la diathèse active (avec ou sans ergatif). Celle à auxiliaire izan a peutêtre, en théorie du moins, un contenu sémantique légèrement différent, l'agent y est moins actif, l'action moins volontaire?

- Dans Hiruak $_{\mathrm{ABS}}$ mintzatzen dira euskaraz ${ }_{\mathrm{INST}}$ on a certes un changement d'auxiliaire, izan « être » pour *edun « avoir », mais contrairement à la définition de Siverstein on n'a pas de suffixe dérivationnel ; contrairement à la voix passive des langues accusatives indo-européennes de la tradition grammaticale, on n'a pas de flexion spécifique du verbe. On a simplement les deux solutions casuelles dont le basque dispose dans le cas de verbes biactanciels en diathèse active non marquée, mais ici avec un même verbe, à savoir Absolutif+Instrumental avec auxiliaire izan « être » et Ergatif + Absolutif avec auxiliaire *edun « avoir ». Dans les exemples Rebuschi et Coyos à sa suite, on a toujours une marque aspectuelle ta (biscayen), $-a$ (central) ou $-i k$ (dialectes orientaux) suffixée au verbe de sens plein, noyau de la phrase, qui accompagne le changement d'auxiliaire par rapport à la construction non marquée ${ }^{8}$. L'intransivation est accompagnée d'une détermination verbale supplémentaire.

- Sur le plan du marquage casuel, par contre, il y a bien un retournement dans le type de Rijk en ce sens que l'argument à rôle sémantique agentif

\footnotetext{
${ }^{6} \mathrm{~J}$ 'ai pour ma part listé les verbes de ce type en dialecte souletin. Ces verbes à variantes de module casuel (ici mintzatü «parler» Absolutif+Instrumental avec auxiliaire izan «être» et Ergatif+Absolutif avec auxiliaire *edün «avoir», mais aussi le type behatü «écouter» Absolutif+Datif et Ergatif + Absolutif, que de Rijk ne signale pas) sont, me semble-t-il, « particulièrement importants dans une perspective typologique et de dynamique de la langue... Ils sont la matérialisation en synchronie d'une passerelle entre deux modèles d'indication de la transitivité... On étendrait un modèle simple, prototypique, l'ergatif pour agent et l'absolutif pour patient. Le degré de transitivité n'a pas changé mais il semblerait pour simplifier qu'on passe d'un couple de rôles sémantiques Expérient-Thème / Localisation au couple Agent-Thème » (Coyos, 1999 : 350).

${ }^{7}$ Dans l'esprit de mes informateurs souletins, le caractère plus passif de la construction en izan n'est pas du tout évident. Ils considèrent ces constructions comme synonymiques.

${ }^{8}$ Considérer que « la paraphrase umea liburua leiuta dao... n'a plus le statut d'une phrase simple : son verbe dao sert de copule et son prédicat liburua leiuta est un prédicat complexe, construit luimême à partir d'une phrase » (de Rijk, 2002 : 299) ne change rien à l'affaire. Cette construction est marquée par rapport à celle type Umeak $k_{\mathrm{ERG}}$ liburua $_{\mathrm{ABS}}$ leiu dau.
} 
matérialisé par le nom à ergatif suffixé, ici hiruek, est matérialisé par un nom à absolutif suffixé, hiruak, dans la deuxième construction. Et conformément à la définition de Silverstein, l'argument à rôle sémantique non agentif est matérialisé par un nom à cas oblique suffixé dans la deuxième construction, ici l'instrumental euskaraz. Ce n'est pas le cas dans les exemples de Rebuschi et Coyos. Certes l'argument à rôle sémantique agentif y est aussi indiqué par un nom à absolutif suffixé ; mais, comme le dit de Rijk, ces exemples ne vérifient pas la définition de Silverstein car l'argument à rôle sémantique non agentif est matérialisé par un nom à cas absolutif suffixé et non un cas oblique. Umea et liburua sont tous les deux à l'absolutif. Cette construction peut être ambiguë hors contexte et situation puisque deux rôles sémantiques bien distincts sont indiqués par un même cas.

- Pour autant il apparaît que l'antipassif type Rebuschi est un parcours diathétique marqué, l'argument non agentif n'étant pas indispensable. En effet « la spécificité de ce parcours est de permettre de qualifier une entité à rôle agentif par un comportement impliquant une autre entité à rôle non agentif, que celle-ci soit matérialisée ou pas dans l'énoncé... De même au passif on qualifie une entité à rôle non agentif à l'aide d'un comportement, l'entité à rôle agentif impliquée dans l'événement n'étant pas systématiquement retenue » (Coyos, 2002 : 299). La dimension aspectuelle de rétrospectif indiquée par $-i k,-a$ ou $-t a$ ne se trouve pas dans la construction active type de Rijk Absolutif+Instrumental avec auxiliaire izan « être " ${ }^{9}$. La construction active en izan ne se distingue pas sur ce plan de celle en *edun.

- La possibilité de ne pas mentionner l'argument à rôle sémantique non agentif pourrait aussi être une des fonctions de l'antipassif type Rebuschi, mais ce n'est pas une spécificité de celle-ci. L'antipassif version de Rijk, mieux nommé diathèse active à auxiliaire izan, autorise aussi cet effacement, du moins avec certains verbes.

En conclusion, il ressort de cette comparaison qu'il paraît plus judicieux de considérer comme relevant d'une diathèse antipassive la construction intransitive avec détermination aspectuelle rétrospective type Umea $a_{\mathrm{ABS}}$ liburua $_{\mathrm{ABS}}$ leiuta dao ou Haurrak $\mathrm{ABS}_{\mathrm{AB}}$ zopa $_{\mathrm{ABS}}$ janik dira, plutôt que celle type Hiruak $_{\mathrm{ABS}}$ mintzatzen dira euskaraz ${ }_{\mathrm{INST}}$ qu'il convient de qualifier de diathèse active à auxiliaire izan.

Quant à la non adéquation avec la définition de Silverstein concernant le cas oblique, il faut savoir que les linguistes anglo-saxons qui ont forgé ce concept travaillaient sur les langues ergatives australiennes ou amérindiennes et qu'ils ignoraient la diversité des parcours diathétiques en basque ; le marquage de deux fonctions syntaxiques par un même indicateur de fonction, ici le cas absolutif, n'est pas non plus un schéma attendu. A leur décharge, on l'a évoqué, les bascologues eux-mêmes ne tenaient pas compte des constructions de type antipassif du basque, celles de type passif étant considérées comme des

\footnotetext{
${ }^{9}$ Dans ses Parameters in the Grammar of Basque, Jon Ortiz de Urbina n'a pas tenu compte de la construction antipassive telle qu'elle est conçue ici; il n'a pas relevé l'existence de telles constructions : «There is no equivalent structure in Basque, although lexically somme verbs appear in two structures that are closely parallel to the structures found in the ergative-antipassive relation » (1989: 203, note 15). Il cite oroitu «se rappeler », c'est-à-dire un verbe à double module casuel (Absolutif+Instrumental / Ergatif+Absolutif) qui fait partie de ceux qu'a retenus ensuite de Rijk.
} 
« pseudo-passifs », des « tours passifs » empruntés aux langues environnantes et ne faisant pas partie de la langue ${ }^{10}$.

\section{QUELQUES OBSERVATIONS A PROPOS DE LA NOTION DE SUJET ET LA LANGUE BASQUE}

\subsection{Sujet : une notion de linguistique générale, ancienne, polysémique, très largement utilisée}

Si le terme antipassif a moins de trente ans, celui de sujet provient des grammaires logiques de l'Antiquité dans lesquelles on distinguait dans la structure prédicative sujet (thème) et prédicat (rhème). «Toute tradition grammaticale est saisie dans un réseau de termes, dans une vision du monde préalable... Du même coup, la linguistique ne peut « emprunter» un terme à une tradition grammaticale, sans aussitôt importer avec lui l'ensemble discursif où il a pris naissance » (Milner, 1989 : 66). Faire un historique du concept de sujet à travers la pensée, les théories linguistiques successives serait un travail utile mais colossal. De même, montrer comment ce terme a été utilisé dans les recherches et les grammaires basques pourrait faire l'objet de plusieurs volumes. Sans entrer dans une étude poussée de ce type, il peut être intéressant d'esquisser en quoi la charge conceptuelle accumulée par ce terme, au cours de son histoire en relation avec les langues accusatives indo-européennes, peut influencer l'usage qui en est fait à l'heure actuelle à propos de la langue basque. On ne trouvera donc pas ici une nouvelle définition de cette notion pour le basque, une nouvelle démonstration cherchant à prouver que c'est telle ou telle unité linguistique ou groupe d'unités qui assume cette fonction dans la phrase basque. Sans aller jusqu'à confronter les modèles théoriques, qui il faut le souligner ont quasiment tous emprunté la notion de sujet pour l'intégrer dans leur appareil conceptuel, il s'agit d'éclaircir un tant soit peu le terrain pour comprendre à quoi chacun fait référence quand il parle de sujet en basque ${ }^{11}$. Il ne s'agit donc pas de rejeter un terme a priori parce qu'il est trop chargé, mais de l'utiliser avec précaution après l'avoir clairement défini dans le modèle théorique mis en œuvre.

La conclusion sera peut-être que chacun, dans son cadre théorique avec son appareil conceptuel et son argumentation, est conséquent quand il fait référence à un sujet en basque. Le tout sera alors de l'admettre et d'accepter que tel autre ne parvienne pas à la même conclusion. Une conséquence serait alors que le concept de sujet ne peut pas faire partie du métalangage de la linguistique générale, du moins sur la base de son usage en bascologie. En effet, malgré sa prudence, Lazard semble optimiste quand il écrit à propos de notions comme « sujet», « objet direct», « transitivité »... : « Dans le meilleur des cas, ces mots peuvent avoir un sens précis quand ils sont appliqués à la grammaire d'une langue

\footnotetext{
${ }^{10}$ Dans « Antipasiboaren lekua oraiko zubereran », j'ai montré qu'en basque souletin parlé et écrit les constructions relevant du parcours diathétique passif étaient fréquentes, celles relevant du parcours diathétique antipassif rares, bien que, selon les locuteurs consultés, ces dernières soient plus conformes à la langue que celles de type passif. On peut supposer qu'en basque la diathèse antipassive est une diathèse plus ancienne.

${ }^{11} \mathrm{Je}$ ne vais pas présenter tous les auteurs et l'usage qu'ils ont fait du terme sujet. Seuls quelquesuns, travaillant dans des cadres théoriques différents, seront succinctement évoqués. En 1978, Georges Rebuschi a mené un travail critique plus technique dans «Cas et fonction sujet en basque » sur les points de vue de l'époque. Il parvenait à la conclusion attendue que « le recours aux concepts de sujet et d'objet reste insuffisant pour analyser les phénomènes liés à la transitivité, à l'ergativité et à la thématisation » $(1978: 97)$.
} 
donnée » (1998: 113); il devrait compléter par ce rajout : " dans le cadre d'un modèle théorique donné »».

La difficulté avec la notion de sujet, c'est qu'elle ressortit dans l'usage qui en est fait, non pas à un mais au moins à trois grands domaines distincts de l'analyse linguistique : indirectement la logique et directement l'énonciation quand on en retient la dimension présentée plus haut, « ce dont on affirme quelque chose ", la sémantique quand on fait référence à « celui qui est dans l'état, fait ou subit l'action qu'indique le verbe », la syntaxe et la morphologie quand on étudie uniquement la fonction grammaticale, les phénomènes d'accord et les autres contraintes liées.

Dans la brève présentation qui suit sera confirmée une des difficultés habituelles rencontrées en linguistique générale, à savoir que si différentes théories peuvent utiliser le même terme, en l'occurrence ici celui de sujet, elles ne lui donnent pas la même signification non seulement parce qu'elles peuvent le définir différemment en référence à un, deux ou trois des domaines cités et en retenant une ou plusieurs propriétés par domaine, mais en plus parce que leur appareillage conceptuel n'est pas le même et n'est pas transposable.

\subsection{Bref aperçu de quelques points de vue sur le sujet en basque}

C'est une histoire bien connue des spécialistes que nous allons parcourir brièvement, de manière informelle. Sa constante, c'est la difficulté à appliquer les critères de définition du sujet de la tradition grammaticale occidentale à une langue à marquage casuel et indiciel globalement ergatif, tout en conservant ce terme de sujet.

Dès le XIXe siècle, la théorie de la passivité du verbe dans les langues ergatives est le premier point de vue non impressionniste sur ce type de langue ${ }^{12}$. Elle s'appuyait de façon partielle sur les formes observables, déterminant nominal à cas dit nominatif/absolutif de forme vide dans les phrases à verbe intransitif comme transitif mais référant alors au patient, agent matérialisé par un nominal avec un cas de forme pleine dit actif / ergatif dans les phrases à verbe transitif, et sur leur traduction dans les langues accusatives indo-européennes. Elle a amené à poser un sujet en basque. Prenons seulement deux exemples. Henri Gavel écrivait: " ces deux fonctions essentielles du nominatif n'en font qu'une en réalité... le mot qui en français serait le complément direct du verbe transitif est en basque le véritable sujet, et le mot qui en français serait le sujet du verbe transitif devient en basque un complément d'agent» (1929, chapitre II : 12). De même C. C. Uhlenbeck pouvait écrire : «ce que nous pourrions apparemment considérer comme sujet grammatical du verbe transitif, est sans doute le sujet logique, mais n'est point du tout le sujet grammatical, et ce que nous prenions pour l'objet passif est plutôt le sujet grammatical» (1948: 73).

Pour sa part, conformément à la tradition grammaticale, Pierre Lafitte, qui s'était opposé à la vision passiviste, plaque les critères de repérage du sujet des langues accusatives, en accord avec le sémantisme : "La difficulté en basque c'est que le sujet de la construction intransitive se met au nominatif, et celui de la conjugaison transitive à l'actif » $(1944: 354, \S 667)$.

\footnotetext{
${ }^{12}$ On omet les travaux antérieurs que Oyharçabal qualifie de « grammaire traditionnelle » (M. de Larramendi 1729 El impossible vencido Arte de la lengua vascongada, F. Lécluse 1828 Manuel de la langue basque) : " Elle ne tient pas compte de la morphologie, et considère simplement qu'il existe deux formes de nominatif pour marquer les sujets... et d'autre part un accusatif pour marquer l'objet » (1993:36).
} 
Dans les années 1960, pour André Martinet et les structuralistes fonctionnalistes, le seul critère de reconnaissance d'un sujet est celui de déterminant obligatoire du prédicat verbal. Influencé par la théorie passiviste, Martinet a nié dans un premier temps l'existence même en basque d'un complexe minimal «sujet-prédicat»: le basque «ignore le syntagme sujet-prédicat et... construit régulièrement ses énoncés par déterminations successives d'un prédicat d'existence »(1958-1974: 215), puis il niait celle de sujet: «En basque, la position par rapport au prédicat n'est pas un trait décisif, puisqu'il n'y a pas de sujet et que la forme à désinence zéro représente une fonction unique » (1962 : 81). Dans le même cadre théorique, Geneviève N'Diaye observait que, en basque, parmi « les compléments qui sont représentés par des indices personnels », celui qui est non-marqué (au cas absolutif) «présente la particularité de figurer dans tous les énoncés à prédicat verbal » $(1970: 59)$. Elle le nomme « complément du premier degré ». Ceci amènera Martinet à poser ensuite l'existence d'un sujet syntaxique en basque, la fonction sujet étant une fonction obligatoire, matérialisée par le déterminant nominal à l'absolutif. « En principe et en fait, la fonction sujet n'a aucune valeur propre », ceci «a du mal à s'imposer... parce que dans les langues les mieux connues... la plupart des nominaux en fonction sujet désignent l'agent du procès » (1985: 178). La notion d'objet n'est pas invoquée, considérer que le déterminant à l'absolutif des phrases à verbe transitif entre dans une fonction objet n'ayant alors pas de sens.

Pour ma part, restant dans un cadre structuraliste fonctionnaliste, face au terme «sujet» bien trop connoté et pour désigner ce seul fait de la présence obligatoire du déterminant d'absolutif, j'ai été amené à parler d'" actualisateur généralisé ", et pour désigner le déterminant d'ergatif, obligatoire avec les seuls verbes transitifs à auxiliaire *edun « avoir », d' « actualisateur non généralisé » $(1999: 292)^{13}$. A ce propos, je tiens à souligner qu'on ne peut tenir pour non pertinent, "phénomène de structure superficiel», simple fait de morphologie, le marquage casuel et indiciel, qu'il soit ergatif, accusatif ou autre. En effet l'indication des relations entre unités significatives est typiquement un phénomène qui relève de la syntaxe dès l'instant où, mettant en relation des unités significatives, nominaux et verbes, cette mise en relation véhicule du sens (repérage des rôles sémantiques respectifs, participation à l'élaboration du contenu du procès). On préférera parler de «marquage casuel ergatif», « marquage indiciel ergatif », plutôt que de " morphologie ergative » qui pourrait laisser entendre qu'il s'agit d'un phénomène sans valeur.

Dans d'autres théories, les notions de «sujet» et d' « objet» sont posées comme des "primitives" et considérées donc comme universelles. On cherche ensuite à attribuer des propriétés syntaxiques et / ou sémantiques et / ou énonciatives à ces deux concepts en fonction de la langue considérée. L'exemple le plus connu est celui de Keenan dans "Towards a Universal Definition of "Subject" » qui propose d'identifier le sujet dans toute phrase de n'importe quelle langue et pour les langues ergatives celui d'Anderson dans «On the Notion of Subject in Ergative Languages ", dans la même publication (1976). Autre exemple avec Dixon pour qui « sujet » est une catégorie universelle, avec trois « universal

\footnotetext{
${ }^{13}$ En termes très généraux l'actualisation est le phénomène qui fait que l'émission sonore perçue est interprétable, qu'elle forme un tout cohérent. En basque la présence d'indice(s) de personne est une condition sine qua non de l'actualisation du noyau syntaxique verbal. On n'évoquera pas ici le débat sur l'existence et la nature d'indices dits de « personne 3 » d'absolutif et d'ergatif ; avec ou sans, la coréférence fonctionne et l'interprétation des énoncés basques est possible.
} 
syntactic-semantic functions »: $S$ « sujet intransitif», $A$ « sujet transitif», $O$ « objet transitif» (1994: 124). Au contraire de la définition martinétienne, ces caractérisations de la notion de sujet sont toutes à critères multiples et relèvent de plusieurs domaines de l'étude des langues.

Karmele Rotaetxe a appliqué la démarche de Keenan au basque dans Estudio estructural..., p. 657 à 670. Sept propriétés du candidat sujet sont étudiées. En bref, notons que ces propriétés que vérifie l'élément à l'ergatif ne sont pas aussi définitoires qu'on pourrait le penser au premier abord ${ }^{14}$. La propriété 1 dite « Equi NP deletion »dans les constructions avec nahi « vouloir» ou les verbes de mouvement n'est pas discriminante car il semble qu'il s'agisse d'un invariant linguistique, la propriété 2 dite « élévation du sujet »est plus liée à un conditionnement sémantique qu'à une propriété syntaxique de l'élément à l'ergatif, la propriété 3 «la formation de la coordination» est liée au fait que l'élément à l'ergatif est généralement thème discursif, la 4 «existence indépendante », " indispensabilité », « référence autonome» est liée à des constantes du flux informatif indépendantes du type syntaxique de la langue, etc. Le sujet est ainsi défini comme un faisceau de propriétés, « ...sujetoa zein den jakiteko problema izaten denean, orduan proprietate gehiago erakutsiko dituen sintagma sujetotzat har daiteke " (1980: 422). Sujet est donc ici une notion relative. « Todas ellas [propriedades] muestran que el ergativo vasco reúne mayor grado de « subjetidad » que el absoluto...el término «absoluto » no indica más que un aspecto formal » (1978: 443-444). Mon point de vue n'est pas éloigné concernant le statut de l'indice d'absolutif et la relativité du caractère subjectif. Mais, dans cette analyse, les trois plans de la syntaxe (actualisation, détermination $\mathrm{du}$ verbe, constructions complexes), de la sémantique (rôle agentif (in)volontaire ou de contrôle, traits sémantiques) et de la visée ou stratégie énonciative (thème, élément focalisé, coréférence, contraintes du flux discursif) sont mêlés pour faire de l'indice d'ergatif ou du nominal correspondant le «sujet» dans les phrases à verbes transitifs à auxiliaire *edun « avoir ». En 1998, sans changer son analyse, K. Rotaetxe conclut : "Le sujet, en basque, a deux expressions morphologiques, $\mathrm{ABS} / \mathrm{ERG}$, en distribution complémentaire » (1998: 870). L'Académie de la langue basque a elle aussi adopté ce point de vue, sans le justifier : "Subjetua absolutuan edo ergatiboan joaten da ; objetua absolutuan edo datiboan... (1991 : 12).

Le modèle génératif, dans ses évolutions successives avec des types de représentation multiples, a été largement appliqué à la langue basque. La définition des notions de sujet et d'objet ne pose pas problème puisqu'on les utilise pour introduire la question: "dans les langues à morphologie casuelle ergative le sujet des phrases intransitives et l'objet des phrases transitives reçoivent la même marque casuelle (cas dit absolutif); le sujet des phrases transitives a une marque différente (cas dit ergatif) » (Oyharçabal, 1993 : 31). On en retiendra que «sujet» comme « objet» relèvent d'abord de la structure profonde ou initiale. Je prendrai ici un seul exemple, Parameters in the Grammar of Basque d'Ortiz de Urbina qui correspond à l'époque de la Théorie du liage

\footnotetext{
${ }^{14}$ Dans Coyos, 1999, j'ai fortement relativisé la portée de certaines d'entre elles et mis en doute la nature syntaxique de quelques-unes. Pour plus de détails se reporter à cet ouvrage. Keenan luimême, en 1984, relativisait les constats faits huit ans plus tôt dans Towards... où, p. 312, il notait d'ailleurs qu'il n'y a pas de combinaison de propriétés nécessaire et suffisante. Dans « Semantic correlates... », il estime qu'il y a aussi une batterie de propriétés que les déterminants à l'absolutif ont en commun (dans la phrase intransitive et transitive) et que le déterminant à l'ergatif n'a pas.
} 
(Government and Binding), n'étant pas à même de discuter la validité des différentes positions au sein du modèle générativiste. "The subject position is (also) obligatory for all levels » (1989: XVI), à savoir structure profonde, de surface et "forme logique" (Logical Form) ; « its obligatoriness... follow(s)... from the requirement that predicates (VP's) be predicated of something, namely, the subject in this case. If the latter is missing, the predicate is not licensed and the structure is ruled out" (ibid.). "subjects c-command objects, but not viceversa" (XVIII). La prise en compte du marquage casuel ergatif du basque est la suivante : le « sujet» des phrases intransitives reçoit le cas absolutif parce qu'il est un objet en «structure profonde» et qu'il «monte» en position sujet en «structure de surface $»^{15}$. Dans ce modèle théorique, on retrouve à nouveau les notions de sujet, d'objet et de prédicat, sujet étant subdivisé de façon traditionnelle, comme le fait Dixon, en intransitive subject (IS) et transitive subject (TS). Et finalement la problématique reste la même ; je cite Ortiz de Urbina en conclusion du chapitre sur l'ergativité en basque : "I have examined what seems to me to be the most crucial question ergative languages pose for linguistic theories : the creation of an asymmetry between core verbal arguments that does not correspond with the subject/ object asymmetry found in accusative languages » (1989: 61).

Revenons, pour terminer ce parcours, à une analyse récente moins formelle des faits de langues. Gilbert Lazard écrit : « La question du sujet est l'une des plus délicates de la grammaire de l'actance... dans les langues européennes [je dirais plutôt accusatives] (où) l'identification du sujet n'est aucunement problématique...: présence obligatoire, position en tête de phrase, marque casuelle zéro, accord du verbe, commande du réfléchi, etc... Mais dans d'autres langues, il n'en va pas de même. Peut-être y en a-t-il où la notion même de sujet n'est pas opératoire » $(1998: 129)$. Lazard propose donc de distinguer deux types de sujets: le «sujet de prédication» qui se caractérise par «la présence obligatoire, le cas zéro et la commande prioritaire de l'accord du verbe, qui sont le propre de l'actant représentant le patient » (1998:129) et le « sujet de référence » caractérisé par «la position initiale et un comportement particulier en cas de coréférence simple ou complexe: ces propriétés s'attachent généralement à l'actant représentant l'agent » (ibid.).

Si dans d'autres modèles théoriques, sujet peut être une notion opératoire, il ne m'appartient pas d'en juger ici, dans le cadre du structuralisme fonctionnaliste et pour une langue à marquage casuel et indiciel globalement ergatif comme la langue basque, la notion de sujet n'est pas utile. La distinction que fait Lazard entre sujet de prédication et sujet de référence, même si elle est fondée sur une analyse que je prends tout à fait à mon compte et que j'ai pu mener dans mon étude du dialecte souletin, me paraît devoir être nommée autrement ${ }^{16}$. Il paraît peu utile de considérer que l'on a deux sujets dans ce type de langues. Il s'agit en

\footnotetext{
${ }^{15} \mathrm{Au}$ contraire, à partir de l'étude des verbes intransitifs dits « inergatifs 》 et «accusatifs » du basque, Oyharçabal conclut : «il convient de prendre en compte l'ambiguité de l'absolutif..., et, en particulier de ne pas associer de manière bi-univoque marquage zéro et objet initial » (1993 : 48).

${ }^{16}$ Dans ce travail, j'ai remis en cause quelque peu certaines des affirmations largement répandues sur l'accusativité syntaxique du basque, en liaison avec les concepts de sujet et de morphologie ergative (1999 : voir la synthèse p. 392-396). J'ai conclu l'analyse en considérant la langue basque dans la forme étudiée comme une langue à pivot peu marqué puisque les traits pouvant être comme considérés comme relevant de l'ergativité ou de l'accusativité ne semblent pas prépondérants (pivot, concept emprunté à Dixon, est présenté plus loin).
} 
effet, face au marquage ergatif, de prendre en compte et les contraintes morphosyntaxiques rappelées ci-dessus et les caractéristiques sémantiques (transitivité, degré de contrôle, lien marquage casuel et rôles sémantiques) et la dimension énonciative (thème, élément focalisé, apport informatif, continuité référentielle), et d'en dégager les conditionnements. Dans cet objectif, la notion de pivot développée par Dixon (1994) me paraît plus opérationnelle et moins connotée, tant pour les langues dites ergatives que les autres types, même si je ne suis pas en accord avec l'ensemble de la terminologie et des concepts qu'il met en oeuvre. Le terme sujet pourrait être conservé pour les langues dans lesquelles justement les propriétés relevant de ces trois domaines distincts seraient assumées par une même unité (nominal déterminant le verbe, argument unique ou à rôle agentif, thème de l'énoncé). Dixon part lui aussi de trois «primitive relations », celles qu'il utilise dans la définition de l'antipassif donnée plus haut en 2.1: "all languages work in terms of... $S$ intransitive subject, $A$ transitive subject, $O$ transitive object » (1994:6). Les langues traitant de façon identique $S$ et $A$ dans les règles de combinaisons de propositions sont dites "S/A pivot», celles traitant de façon identique $S$ et $P$ sont dites « $\mathrm{S} / \mathrm{O}$ pivot» (11). $A, S$ et $O$ sont des « universal syntactic-semantic functions » (124), ce qui est moins clair et rappelle la difficulté évoquée ci-dessus à dissocier syntaxe et sémantique. "Only some languages work in terms of a pivot, and this only these that can be characterised as 'accusative' or 'ergative'... (144). Où la réflexion devient encore plus intéressante, c'est quand Dixon convient que pour expliquer certains faits il faut dépasser le strict cadre morphologique et syntaxique: «It may be that we need to look beyond syntax, to discourse structure» (177). Ainsi en fin d'ouvrage, Dixon prend toute la mesure de la problématique posée par la caractérisation typologique d'une langue : « The most important task for future work on 'why some languages are ergative in a certain way and others are not' is to investigate the semantic and discourse-pragmatic make-up of each of a sample of languages, and study the way in which this determines (or partly determines) its grammatical profile" (219-200). Dans cette perspective la tâche reste grande, même pour une langue « moyennement » décrite comme le basque. Des études partielles toujours plus nombreuses sont menées, mais il manque sûrement une synthèse prenant en compte toutes les dimensions de l'analyse linguistique et mettant à jour leur interdépendance, l'interaction entre les domaines.

\section{EN GUISE DE CONCLUSION}

Ici il me paraît utile de renvoyer le lecteur à l'article de Gilbert Lazard dans l'esprit duquel s'inscrit ce travail, Pour une terminologie rigoureuse : quelques principes et propositions, en le citant une dernière fois: "Les catégories de langues particulières sont définissables rigoureusement... Les catégories « interlangues »... sont des notions floues, qui parlent à l'intuition, mais restent mal définies parce qu'elles réfèrent à des catégories de langues particulières, catégories qui ont bien quelque chose en commun, mais qui couvrent des ensembles différents de sens et d'emplois » (1998:116). Ceci ne signifie pas qu'une théorie linguistique ne peut pas avoir son appareillage conceptuel, ses principes, sa propre terminologie. Les catégories en linguistique générale sont d'une autre nature, toute la difficulté étant de dégager ce qui est commun aux 
langues, les invariants, de ce qui est particulier ou en tout cas décliné de façon particulière dans la langue considérée.

\section{BIBLIOGRAPHIE}

ANDERSON Stephen R., 1976, On the Notion of Subject in Ergative Languages, Subject and Topic, Charles N. Li ed., New York, Academic Press, p. 1-23.

CoYos Jean-Baptiste / Battittu, 1999, Le parler basque souletin des Arbailles Une approche de l'ergativité, Paris, L'Harmattan, $432 \mathrm{p}$. ,2002, Parcours de type passif et de type antipassif en basque souletin parlé actuel, Bulletin de la Société de Linguistique de Paris, XCVII, fasc. 1, Paris - Louvain, Peeters, p. 281-314.

2003, Antipasiboaren lekua oraiko zubereran, Lafitte sortzearen mendemuga, Euskaltzaindiaren nazioarteko XV. Biltzarra, Baiona, $2001 /$ 09 / 20-21, Iker, 14, I, Bilbo, Euskaltzaindia, 181-193.

DiXON Robert M. W., 1994, Ergativity, Cambridge, Cambridge University Press, $\mathrm{xxii}+271 \mathrm{p}$.

EUSKALTZAINDIA, Gramatika batzordea, 1991, Euskal gramatika Lehen urratsak-I, Bilbo, Euskaltzaindia / R.A.L.V., XXX+569 p.

Gavel Henri, 1929, Grammaire basque, Tome I : Phonétique - Parties du discours autres que le verbe, Bayonne, 69 et $221 \mathrm{p}$.

KeEnAN Edward L., 1976, Towards a Universal Definition of "Subject", Subject and Topic, Charles N. Li ed., New York, Academic Press, p. 303333.

1984, Semantics correlates of the ergative / absolutive distinction, Linguistics, 22, 2, Berlin - New York - Amsterdam, Mouton Publishers, p. 197-223.

LAFITTE Pierre, 1944 (1962 / 1991), Grammaire basque (Navarro-labourdin littéraire), Édition revue et corrigée, Bayonne, Elkar-Ikas, 490 p.

LARRASQUET Jean, 1934, Le Basque Souletin Nord-Oriental, tome I, Introduction, Paris, chez l'auteur, $231 \mathrm{p}$.

LAZARD Gilbert, 1998, Pour une terminologie rigoureuse : quelques principes et propositions, La terminologie linguistique, Mémoires de la Société de Linguistique de Paris, tome VI, Paris - Louvain, Peeters, p. 111-133. , 2000, Que cherchent les chercheurs?, Bulletin de la Société de Linguistique de Paris, XCV, fasc. 1, Paris - Louvain, Peeters, p. 445-460. , 2001, De l'objectivité en linguistique, Bulletin de la Société de Linguistique de Paris, XCVI, fasc. 1, Paris - Louvain, Peeters, p. 9-22.

MARTINET André, 1958, La construction ergative et les structures élémentaires de l'énoncé, Journal de psychologie normale et pathologique, juilletseptembre, 1965 (1974), La linguistique synchronique, Paris, P.U.F. , p. 211-227.

, 1962, Le sujet comme fonction linguistique et l'analyse syntaxique du basque, Bulletin de la Société de Linguistique de Paris, 57, Paris, Klincksieck, p. 73-82. , 1985, Syntaxe générale, Paris, A. Colin, 266 p.

MiLNER Jean-Claude, 1989, Introduction à une science du langage, Paris, Seuil, $711 \mathrm{p}$. 
N'DiaYe Geneviève, 1970, Structure du dialecte basque de Maya, The Hague / Paris, Mouton, $249 \mathrm{p}$.

OrtiZ DE Urbina Jon, 1989, Parameters in the Grammar of Basque, Dordrecht Holland / Providence RI - U.S.A., Foris Publications, XX+278 p.

OyHARÇABAL Bernard, 1993, Notion d'objet et cas absolutif en basque, Le Gré des Langues, 5, Paris, L'Harmattan, p. 30-49.

Pollock Jean-Yves, 1997, Langage et cognition. Introduction au programme minimaliste de la grammaire générative, Paris, Presses Universitaires de France, XXII + $241 \mathrm{p}$.

REBUSCHI Georges, 1978, Cas et fonction sujet en basque, Verbum, I, fasc.1, Nancy, p. 69-98.

, 1979, Autour du passif et de l'antipassif en basque biscayen (sous-dialecte d'Oñate), Relations prédicat-actants dans les langues de types divers II, Paris, Selaf, p. 149-170.

RIJK Rudolf P. G. de, 2002, L'antipassif et l'hypothèse de Levin, Lapurdum, VII, Bayonne, Centre de Recherche sur la Langue et les Textes Basques IKER UMR 5478, 295-312.

RotAeTXE Karmele, 1978, Estudio estructural del euskara de Ondárroa, Durango (Vizcaya), Leopoldo Zugaza, 715 p.

1978, Lingüística-Lógica : La construcción ergativa vasca, Revista española de Lingüística, año 8, fasc. 2, Madrid, Gredos, p. 431-445. 1980, Ergatiboaren sujetotasunaz, Euskera, XXV, 2, Bilbo, Euskaltzaindia, p. 417-424.

, 1998, L'actance en basque, Actance et Valence dans les langues de l'Europe, EALT / EUROTYP, 20-2, Jack Feuillet (éditeur), Berlin / NewYork, Mouton - de Gruyter, p. 849-876.

UHLENBECK Christianus Cornelius, 1948, La langue basque et la linguistique générale, Lingua, 1, Amsterdam, North-Holland Publishing Company, p. 59-76. 